\title{
A Road Map of Interval Temporal Logics and Duration Calculi
}

\author{
Valentin Goranko* \\ Angelo Montanari*** \\ Guido Sciavicco $^{* *}$ \\ * Department of Mathematics and Statistics, \\ Rand Afrikaans University (South Africa) \\ vfg@rau.ac.za \\ ** Dipartimento di Matematica e Informatica, \\ Università di Udine (Italy) \\ \{montana,sciavicc\}@dimi.uniud.it
}

ABSTRACT. We survey main developments, results, and open problems on interval temporal logics and duration calculi. We present various formal systems studied in the literature and discuss their distinctive features, emphasizing on expressiveness, axiomatic systems, and (un)decidability results.

KEYWORDS: interval temporal logic, duration calculus, expressiveness, axiomatic system, decidability.

\section{Introduction}

Philosophy. 


\section{Linguistics.}

\section{Artificial intelligence.}

expert systems planning systems theories of actions and change natural language analysis and processing

meets

\section{Computer science.}

duration calculi 
locality

\section{Preliminaries}

2.1. Temporal ontologies, interval structures and relations between intervals

linear

branching? Discrete dense? With without beginning?

Should intervals include their end-points or not?

Can they be unbounded?

Are point-intervals (i.e. with coinciding endpoints) admissible or not?

How are points and intervals related? Which is the primary concept? Should an interval be identified with the set of points in it, or there is more into it?

$$
\begin{aligned}
& \mathbb{D}=\left\langle D,<\quad \text { interval } \quad \mathbb{D} \quad\left[d_{0}, d_{1}\right]\right. \\
& d_{0}, d_{1} \in D \\
& d_{0} \leq d_{1}\left[d_{0}, d_{1}\right] \quad \text { strict } \\
& d_{0}<d_{1} \\
& \mathbb{D} \text { non-strict intervals } \\
& {[d, d]} \\
& \text { point-intervals } \\
& d \text { belongs to an interval }\left[d_{0}, d_{1}\right] \\
& d_{0} \leq d \leq d_{1} \\
& \mathbb{D} \quad \mathbf{I}(\mathbb{D})^{+} \\
& \langle\mathbb{D}, \mathbf{I}(\mathbb{D}) \quad \text { interval structure }
\end{aligned}
$$


linear,

linear interval property:

$x y\left(x<y \rightarrow z_{1} \quad z_{2}\left(x<z_{1}<y \wedge x<z_{2}<y \rightarrow z_{1}<z_{2} \vee z_{1}=z_{2} \vee z_{2}<z_{1}\right)\right)$,

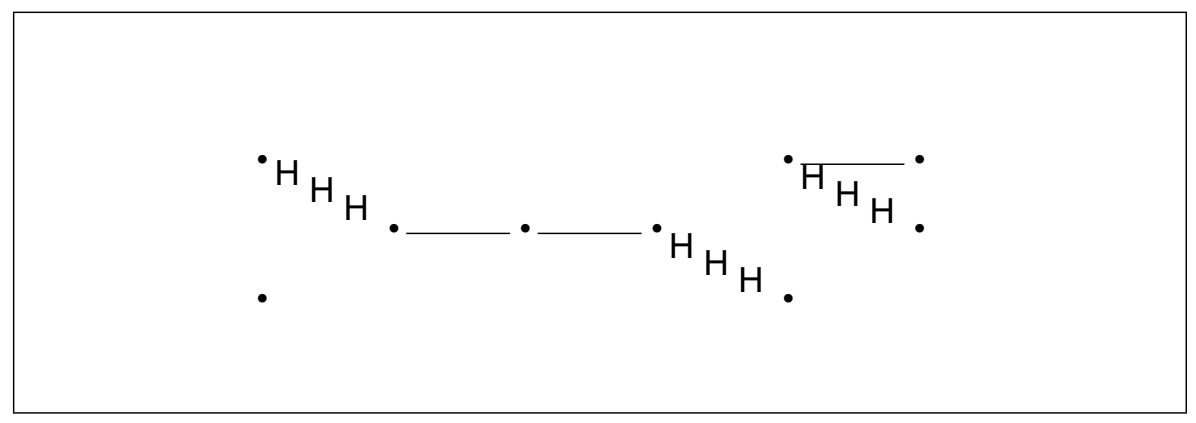

Figure 1. Interval structure with the linear interval property

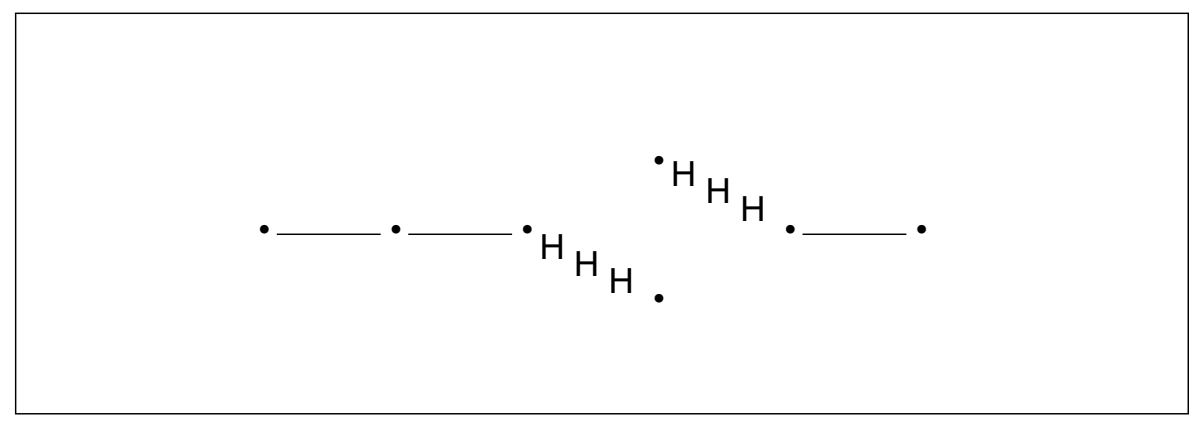

Figure 2. Interval structure violating the linear interval property
linear
discrete
$x y(x<y \rightarrow \exists z(x<z \wedge z \leq y \wedge w(x<w \wedge w \leq y \rightarrow z \leq w)))$
$x y(x<y \rightarrow \exists z(x \leq z \wedge z<y \wedge w(x \leq w \wedge w<y \rightarrow w \leq z)))$ 
dense

$x \quad y(x<y \rightarrow \exists z(x<z \wedge z<y)) ;$

unbounded above below

Dedekind complete

$\mathbb{N}, \mathbb{Z}, \mathbb{Q} \quad \mathbb{R}$

during, begins, overlaps, meets, before

equals, ends,

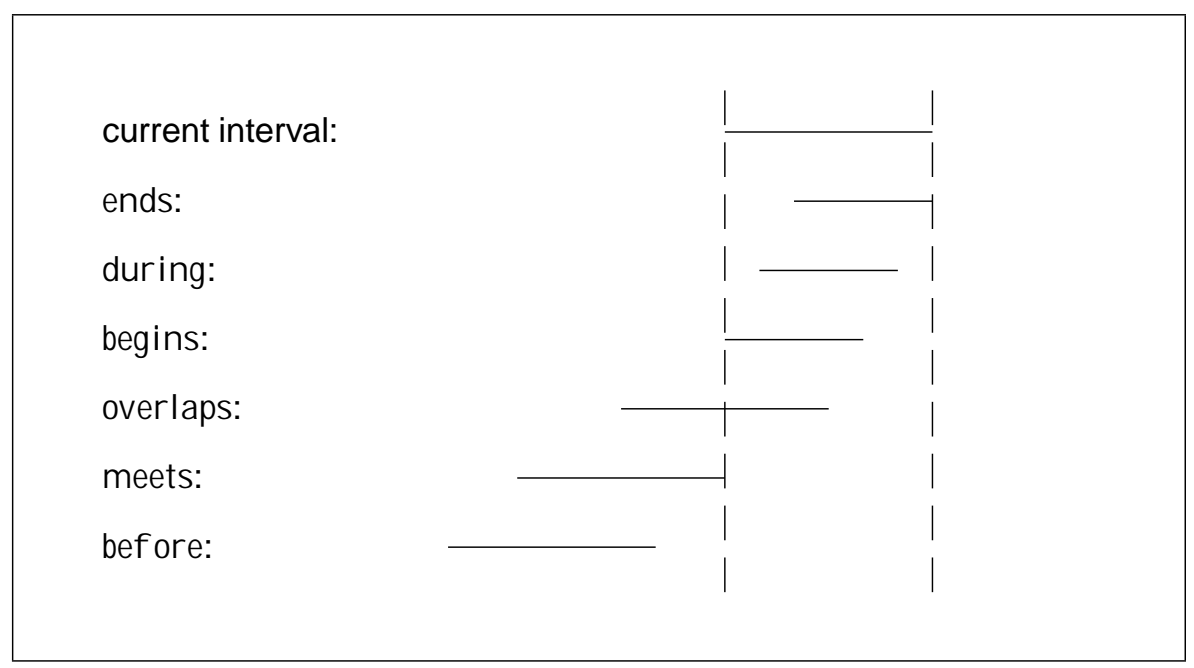

Figure 3. Allen's relations

$$
\begin{gathered}
\text { sub-interval } \\
\mathbb{D}\left[s_{0}, s_{1}\right] \quad\left[d_{0}, d_{1}\right] \\
{\left[s_{0}, s_{1}\right] \quad \text { sub-interval }\left[d_{0}, d_{1}\right] \quad d_{0} \leq s_{0} \quad s_{1} \leq d_{1} .}
\end{gathered}
$$




$$
\begin{array}{cccccc}
{\left[s_{0}, s_{1}\right]} & \text { proper sub-interval } & {\left[d_{0}, d_{1}\right]} & {\left[s_{0}, s_{1}\left\lceil\left[d_{0}, d_{1}\right],\right.\right.} & {\left[s_{0}, s_{1}\right]} \\
{\left[d_{0}, d_{1}\right]\left[s_{0}, s_{1}\right] \neq\left[d_{0}, d_{1}\right]} & & & \\
{\left[s_{0}, s_{1}\right]} & \text { strict sub-interval } & {\left[d_{0}, d_{1}\right]} & {\left[s_{0}, s_{1}\right]} & {\left[d_{0}, d_{1}\right],} & d_{0}<s_{0} \\
s_{1}<d_{1} & \text { ternary } & &
\end{array}
$$

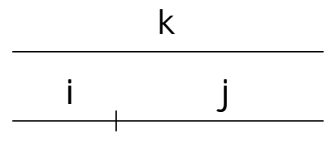

Figure 4. The ternary relation $A$

A

Aijk $i$ meets $j, i$ begins $k$, and $j$ ends $k$

$k \quad i \quad j$

\subsection{Propositional interval temporal languages and models}

$\mathcal{A P}$

poral operators (modalities)

$\begin{array}{ccc}\text { strict interval model } & \begin{array}{c}\text { non-strict } \\ \mathbf{I}(\mathbb{D})^{+} \rightarrow \mathbf{P}(\mathcal{A} \mathcal{P})\end{array} & \mathbf{M}^{+}=\langle\mathbb{D}, V \\ & \text { valuation } & \mathbb{D} \\ & V: \mathbf{I}(\mathbb{D})^{-} & \text {strict interval model } \\ \mathbf{M} & \rightarrow \mathbf{P}(\mathcal{A P}) .\end{array}$

$\wedge$

interval tem-

strict non$V$ : $\mathbf{M}^{-}=\langle\mathbb{D}, V$

begins, ends

$$
\phi::=p|\quad \phi| \phi \wedge \psi \mid\langle B \phi|\langle E \phi|\langle\bar{B} \phi|\langle\bar{E} \phi .
$$




$$
\begin{array}{lllllll}
\left(\langle B) \mathbf{M}^{+},\left[d_{0}, d_{1}\right]\right. & \langle B \phi & \mathbf{M}^{+},\left[d_{0}, d_{2}\right] & \phi & d_{2} & d_{0} \leq d_{2}<d_{1} \\
\left(\langle E) \mathbf{M}^{+},\left[d_{0}, d_{1}\right]\right. & \langle E \phi & \mathbf{M}^{+},\left[d_{2}, d_{1}\right] & \phi & d_{2} & d_{0}<d_{2} \leq d_{1} \\
\left(\langle\bar{B}) \mathbf{M}^{+},\left[d_{0}, d_{1}\right]\right. & \langle\bar{B} \phi & \mathbf{M}^{+},\left[d_{0}, d_{2}\right] & \phi & d_{2} & d_{1}<d_{2} \\
\left(\langle\bar{E}) \mathbf{M}^{+},\left[d_{0}, d_{1}\right]\right. & \langle\bar{E} \phi & \mathbf{M}^{+},\left[d_{2}, d_{1}\right] & \phi & d_{2} & d_{2}<d_{0}
\end{array}
$$

\section{modal constant $\pi$ for point-intervals}

( $\pi) \mathbf{M}^{+},\left[d_{0}, d_{1}\right] \quad \pi \quad d_{0}=d_{1}$

$\pi \quad \pi \quad[B] \perp \quad[E] \perp$

$$
\begin{aligned}
& \tau(p)=p \quad p \in \mathcal{A P} ; \\
& \tau(\phi)=\quad \tau(\phi) \\
& \tau(\phi \wedge \psi)=\tau(\phi) \wedge \tau(\psi) ; \\
& \tau(\langle * \phi)=\langle *(\pi \wedge \tau(\phi))
\end{aligned}
$$

$\phi:$

formula $\phi$ :

For every interval model $\mathbf{M}$, proper interval $\left[d_{0}, d_{1}\right]$ in $\mathbf{M}$, and

$$
\mathbf{M}^{-},\left[d_{0}, d_{1}\right] \quad \phi \text { iff } \mathbf{M}^{+},\left[d_{0}, d_{1}\right] \quad \tau(\phi) \text {. }
$$

A

$$
C, D \quad T
$$

(C) $\mathbf{M}^{+}, k \quad \phi C \psi$

$\mathbf{M}^{+}, j \quad \psi$

$i, j \quad A i j k \quad \mathbf{M}^{+}, i \quad \phi$

$\mathbf{M}^{+},\left[d_{0}, d_{1}\right]$

$\phi C \psi \quad \mathbf{M}^{+},\left[d_{0}, d_{2}\right]$

$d_{2} \in \mathbb{D}$

$d_{0} \leq d_{2} \leq d_{1}$

(D) $\mathbf{M}^{+}, j \quad \phi D \psi$

$i, k$

$\mathbf{M}^{+},\left[d_{2}, d_{1}\right] \quad \psi$

$\mathbf{M}^{+}, k \quad \psi$

$\mathbf{M}^{+},\left[d_{0}, d_{1}\right]$

$\phi D$

$\phi D \psi \quad \mathbf{M}^{+},\left[d_{2}, d_{0}\right] \quad \phi$

$d_{2} \in \mathbb{D} \quad d_{2} \leq d_{0}$

(T) $\mathbf{M}^{+}, i \quad \phi T \psi$

$\mathbf{M}^{+}, k \quad \psi$

j,k Aijk $\quad \mathbf{M}^{+}, j \quad \phi$

$\mathbf{M}^{+},\left[d_{0}, d_{1}\right]$

$d_{2} \in \mathbb{D}$

$$
\phi T
$$

$d_{1} \leq d_{2}$

$\mathbf{M}^{+},\left[d_{0}, d_{2}\right] \quad \psi$

Aijk $\quad \mathbf{M}^{+}, i \quad \phi$

$\mathbf{M}^{+},\left[d_{2}, d_{1}\right] \quad \psi$ 
locality

homogeneity

split-structures

2.3. First-order languages and models for interval logics and duration calculi.

terms

global rigid

temporal flexible

$$
p, q, \ldots \quad i, j \ldots
$$

$$
\phi::=p\left(\theta_{1}, \ldots, \theta\right)|\exists x \phi| \quad \phi \mid \phi \wedge \psi,
$$

$\theta_{1}, \ldots, \theta$

global

$l \quad$ length of the current interval

state expressions 
3. Propositional Interval Logics

non-strict

3.1. Monadic interval logics

The sub-interval logic D

$$
\phi::=p|\phi| \phi \wedge \psi \mid\langle D \phi
$$

$\pi$ $\begin{array}{cccc}\left(\langle D) \underset{\mathbf{M},}{\mathbf{M}}\left[d_{0}, d_{1}\right]\right. & \langle D \phi & {\left[d_{2}, d_{3}\right]} & {\left[d_{0}, d_{1}\right]} \\ \mathbf{M},\left[d_{2}, d_{3}\right] & \phi\end{array}$

$$
\bigwedge_{=1}^{()}\left\langle D\left(p \wedge \bigwedge_{G} p\right) \rightarrow\langle D \quad \top,\right.
$$

$d(n) \quad\left(\begin{array}{c}2 \\ 2\end{array}\right)+1$ 
The logics $\mathrm{B} \overline{\mathrm{B}}$ and $\mathrm{E} \overline{\mathrm{E}}$

single

pairs

$\mathrm{B} \overline{\mathrm{B}}$

$B \bar{B} \quad E \bar{E}$

$$
\phi::=p|\quad \phi| \phi \wedge \psi \mid\langle B \phi|\langle\bar{B} \phi,
$$

$\bar{E}$

$\overline{\mathrm{B}}$

$\mathrm{B} \overline{\mathrm{B}}$

$\langle E \quad\langle B \quad\langle\bar{E} \quad\langle\bar{B}$

$E \bar{E}$

$\mathrm{B} \overline{\mathrm{B}}$

F

P

$$
f::=p|f| f \wedge g|P f| F f,
$$

$\mathcal{V}: \mathbb{D} \mapsto \mathbf{P}(\mathcal{A P})$

$$
\langle\mathbb{D}, \mathcal{V} \quad \mathbb{D}=\langle D,<
$$

$M, d \quad p \quad p \in \mathcal{V}(d)$

$M, d \quad f \quad M, d \quad f$

$M, d \quad f \wedge g \quad M, d \quad f \quad M, d \quad g$

$M, d \quad P f \quad d^{\prime} \quad d^{\prime}<d \quad M, d^{\prime} \quad f$

$M, d \quad F f \quad d^{\prime} \quad d<d^{\prime} \quad M, d^{\prime} \quad f$

$\tau$

$\mathrm{B} \overline{\mathrm{B}}$

and formula $\phi \in \mathrm{B} \overline{\mathrm{B}}$ :

For every model $\mathbf{M}=\langle\mathbb{D}, V$ of $\mathrm{B} \overline{\mathrm{B}}$, with $\mathbb{D}=\langle D,<$, point $d \in D$,

$$
\mathbf{M},\left[d, d^{\prime}\right] \quad \phi \text { iff } \mathbf{M}[d), d^{\prime} \quad \tau(\phi)
$$

for any $d^{\prime} d$. 


$$
\begin{aligned}
& \phi=\left\langle B \psi \quad \tau(\phi)=P \tau(\psi) \quad \mathbf{M},\left[d, d^{\prime}\right]\right. \\
& \left\langle B \psi \quad d^{\prime \prime} \quad d \leq d^{\prime \prime}<d^{\prime} \quad \mathbf{M},\left[d, d^{\prime \prime}\right] \quad \psi\right. \\
& \phi=\langle\bar{B} \psi \\
& \mathbf{M}[d), d^{\prime \prime} \quad \tau(\psi) \quad \mathbf{M}[d), d^{\prime} \quad \operatorname{P} \tau(\psi)
\end{aligned}
$$

A formula $\phi \in \mathrm{B} \overline{\mathrm{B}}$ is satisfiable in a model $\mathrm{M}$ of $\mathrm{B} \overline{\mathrm{B}}$ iff $\tau(\phi)$ is satisfiable in some model $\mathbf{M}[d)$.

$L$

${ }^{+} C=\left\{{ }^{+} L \mid L \in C\right\}$

The satisfiability problem for the logic $\mathrm{B} \overline{\mathrm{B}}$, interpreted in a given class of interval structures over a class of linear orderings $C$, is reducible to the satisfiability problem for the logic interpreted over the class ${ }^{+} \mathrm{C}$.

$\mathrm{B} \overline{\mathrm{B}}$

The logic $\mathrm{BE}$

$$
\begin{array}{r}
\langle B \quad\langle E \\
\phi::=p|\phi| \phi \wedge \psi \mid\langle B \phi|\langle E \phi .
\end{array}
$$

$\pi$ $[B] \perp$

$[[B P]] \phi \quad(\phi \wedge \pi) \vee\langle B(\phi \wedge \pi)$

$[[E P]] \phi \quad(\phi \wedge \pi) \vee\langle E(\phi \wedge \pi)$

$\langle D$

$\langle D \phi \quad\langle B\langle E \phi$

$$
\left\langle\mathbb{I}(\mathbb{D})^{+}, \quad, V\right.
$$

$\mathbb{D}$

$$
\begin{array}{cc}
\langle B & \langle E \\
& \mathbb{I}(\mathbb{D})^{+}
\end{array}
$$

$\mathbb{I}(\mathbb{D})^{+} \quad V$

$$
\begin{aligned}
& d_{0}<d_{1} \quad \mathbb{D}^{\prime}=\left\{d_{0}^{\prime}\right\} \\
& , V \quad \mathbf{M}^{+}=\left\langle\mathbb{I}^{\prime}\left(\mathbb{D}^{\prime}\right)^{+}, \quad, V^{\prime}\right. \\
& \left.\mathbb{I}(\mathbb{D})^{+}=\left\{\left[d_{0}, d_{0}\right],\left[d_{1}, d_{1}\right],\left[d_{0}, d_{1}\right]\right]\right\} \quad \mathbb{I}^{\prime}\left(\mathbb{D}^{\prime}\right)^{+}=\left\{\left[d_{0}^{\prime}, d_{0}^{\prime}\right]\right\} \\
& R \quad \mathbb{D} \times \mathbb{D}^{\prime} \\
& \left\{\left(d_{0}, d_{0}^{\prime}\right),\left(d_{1}, d_{0}^{\prime}\right)\right\} \\
& \{p\}
\end{aligned}
$$$$
\mathbb{D}=\left\{d_{0}, d_{1}\right\}
$$$$
\mathbf{M}^{+}=\left\langle\mathbb{I}(\mathbb{D})^{+}\right. \text {, }
$$ 


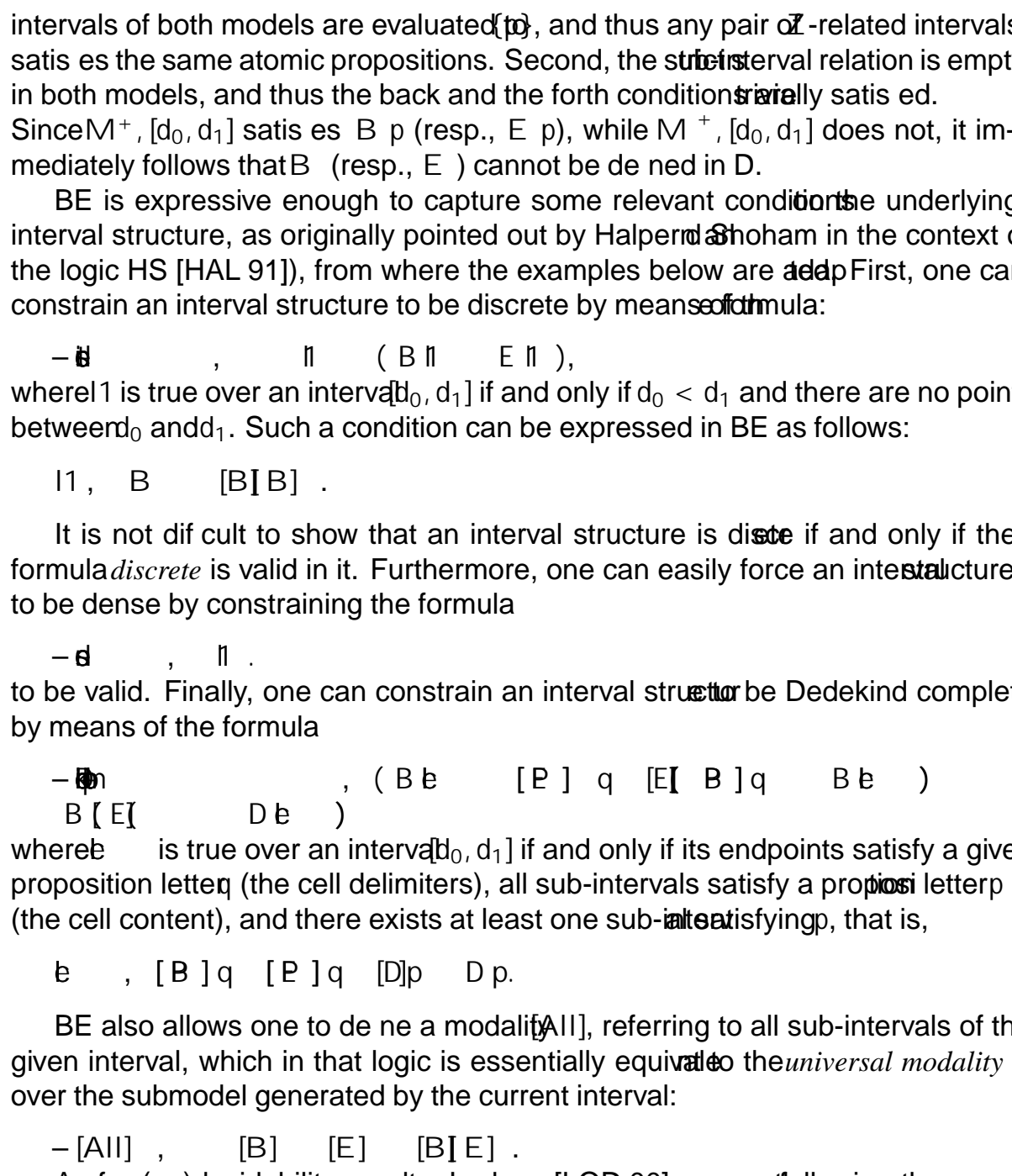

The satisfiability problem for BE-formulas interpreted over nonstrict dense linear structures is not decidable. 


\section{The satisfiability problem for over the class of all non-strict linear structures is not decidable.}

$[A l l] l 1 \wedge \phi$

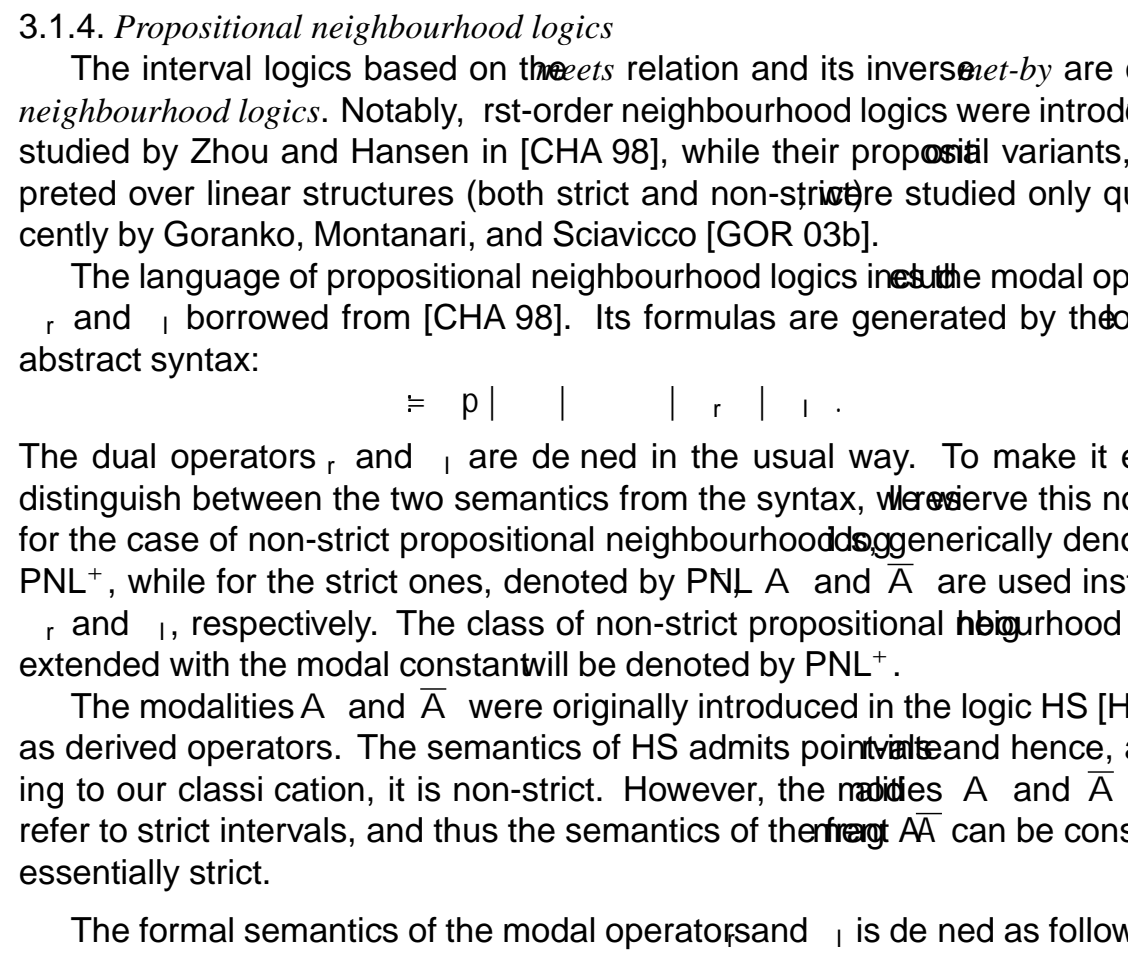




\begin{tabular}{|c|c|c|c|c|}
\hline$(\diamond) \mathbf{M}^{+},\left[d_{0}, d_{1}\right]$ & $\diamond \phi$ & $d_{2}$ & $d_{1} \leq d_{2}$ & $\mathbf{M}^{+},\left[d_{1}, d_{2}\right] \quad \phi$ \\
\hline$(\diamond) \mathbf{M}^{+},\left[d_{0}, d_{1}\right]$ & $\diamond \phi$ & $d_{2}$ & $d_{2} \leq d_{0}$ & $\mathbf{M}^{+},\left[d_{2}, d_{0}\right]$ \\
\hline & & $\langle A$ & $\langle\bar{A}$ & \\
\hline$\left\langle A{ }_{\phi} \underset{\mathbf{M}^{-},\left[d_{0}, d_{1}\right]}{\mathbf{S}^{\prime}}\right.$ & $\langle A \phi$ & $d_{2}$ & $d_{1}<d_{2}$ & $\mathbf{M}^{-},\left[d_{1}, d_{2}\right]$ \\
\hline $\bar{A}) \mathbf{M}^{-},\left[d_{0}, d_{1}\right]$ & $\langle\bar{A} \phi$ & $d_{2}$ & $d_{2}<d_{0}$ & $\mathbf{M}^{-},\left[d_{2}, d_{0}\right]$ \\
\hline
\end{tabular}

(A-SPNL ) $[A] p \rightarrow\langle A p$

unbounded

(A-SPNL ) $(\langle A\langle A p \rightarrow\langle A\langle A\langle A p) \wedge(\langle A[A] p \rightarrow\langle A\langle A[A] p)$

dense

(A-SPNL ) $([A] \perp \rightarrow[\bar{A}]([A][A] \perp \vee\langle A(\langle A \top \wedge[A][A] \perp))) \wedge((\langle A \top \wedge[A](p \wedge$

$[\bar{A}] p \wedge[A] p)) \rightarrow[\bar{A}][\bar{A}]\langle A(\langle A \quad p \wedge[A][A] p))$

discrete

(A-SPNL ) $\langle A\langle A[\bar{A}] p \wedge\langle A[A][\bar{A}] p \rightarrow\langle A(\langle A[\bar{A}][\bar{A}] p \wedge[A]\langle A \quad[\bar{A}] p)$

Dedekind complete

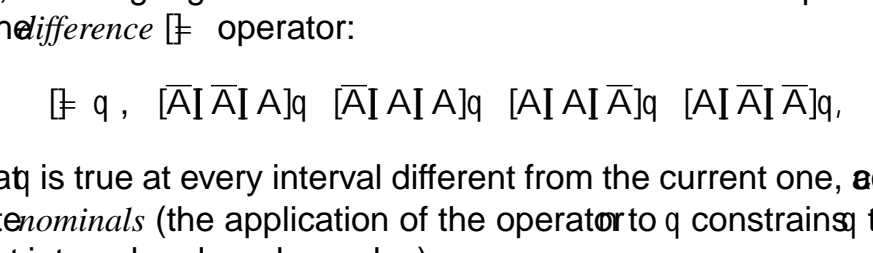

$[\neq] q \quad[\bar{A}][\bar{A}][A] q \wedge[\bar{A}][A][A] q \wedge[A][A][\bar{A}] q \wedge[A][\bar{A}][\bar{A}] q$,

$q$

nominals $\quad n \quad q \quad q$

$n(q) \quad q \wedge[\neq](q)$.

The following axiomatic system is sound and complete for the logic + of non-strict linear structures:

(A-NT)

1 
(A-NK) $\quad K \quad 2 \quad 2$

(A-NNF0) $2 p \rightarrow \diamond p$

(A-NNF1) $p \rightarrow 2 \diamond p$

(A-NNF2) $\diamond \diamond p \rightarrow 2 \diamond p$

(A-NNF3) $2 \diamond p \rightarrow \diamond \diamond \diamond p \vee \diamond \diamond \diamond p$

(A-NNF4) $\diamond \diamond \diamond p \rightarrow \diamond \diamond p$

$\left(\mathbf{A}-\mathbf{N N F}_{\infty}\right) 2 q \wedge \diamond p_{1} \wedge \ldots \wedge \diamond p \rightarrow \diamond\left(2 q \wedge \diamond p_{1} \wedge \ldots \wedge \diamond p\right)$

$n \quad 1$

22

[GOR 03b] A sound and complete axiomatic system for the logic

+ can be obtained from that for $\quad+$ by adding the following axioms:

(A- $\pi \mathbf{1}) \diamond \pi \wedge \diamond \pi$;

$(\mathbf{A}-\pi 2) \diamond(\pi \wedge p) \rightarrow 2 \quad(\pi \rightarrow p)$, and its inverse;

(A- $\pi 3) \diamond p \wedge 2 q \rightarrow \diamond(\pi \wedge \diamond p \wedge 2 q)$, and its inverse.

$\diamond, \diamond \quad\langle A,\langle\bar{A} \quad 2,2$

[GOR 03b] The following axiomatic system is sound and complete for the logic - of strict linear models:

(A-ST) enough propositional tautologies;

(A-SK) the $K$ axioms for $[A]$ and $[\bar{A}]$;

(A-SNF1) $p \rightarrow[A]\langle\bar{A} p$, and its inverse;

(A-SNF2) $\langle A\langle\bar{A} p \rightarrow[A]\langle\bar{A} p$, and its inverse;

(A-SNF3) $(\langle\bar{A}\langle\bar{A} \top \wedge\langle A\langle\bar{A} p) \rightarrow p \vee\langle\bar{A}\langle A\langle A p \vee\langle\bar{A}\langle\bar{A}\langle A$, and its inverse;

(A-SNF4) $\langle A\langle A\langle A p \rightarrow\langle A\langle A p$, and its inverse.

- $\quad \lambda \in\{u, d e, d i, c, u d e, u d i, u c\}$

[GOR 03b] The following hold: 
1) For every $\lambda_{1}, \lambda_{2} \in\{u$, de, di, c, ude, udi, uc $\}, \quad 1^{-} \varsubsetneqq \quad 2^{-}$iff the class of linear orders characterized by the condition $\lambda_{2}$ is strictly contained in the class of linear orders characterized by the condition $\lambda_{1}$.

2) $\quad-\varsubsetneqq \quad+$, where the inclusion is in terms of the obvious translation between the two languages (which replaces the strict modalities with the non-strict ones, and vice versa).

$3)$

$$
\begin{aligned}
& +=\quad+=\quad+=\quad+=\quad+\text {. } \\
& \langle A p \wedge[A](p \rightarrow\langle A p) \wedge[A][A](p \rightarrow\langle A p) \rightarrow[A]\langle A\langle A p \\
& \mathbb{Z}
\end{aligned}
$$$$
-
$$

$$
\mathbb{Z}+\mathbb{Z}
$$

[GOR 03b] The axiomatic system for $\quad-$ extended with (
) (resp. (
), (
), (
), and (
)) is sound

and complete for the class of unbounded (resp. dense, discrete, dense unbounded, and discrete unbounded) structures.

The logic $\mathrm{HS}$

$\langle B,\langle E$

$\langle\bar{B},\langle\bar{E}$

$$
\phi::=p|\quad \phi| \phi \wedge \psi \mid\langle B \phi|\langle E \phi|\langle\bar{B} \phi|\langle\bar{E} \phi
$$

$\langle A \quad\langle\bar{A}$

$$
\begin{array}{ll}
\langle A \phi & {[[E P]]\langle\bar{B} \phi} \\
\langle\bar{A} \phi & {[[B P]]\langle\bar{E} \phi}
\end{array}
$$

I i near

$(\langle A p \rightarrow[A](p \vee\langle B p \vee\langle\bar{B} p)) \wedge(\langle\bar{A} p \rightarrow[\bar{A}](p \vee\langle E p \vee\langle\bar{E} p))$ 


$$
d_{0}, d_{1}, d_{2}, \ldots \quad d<d_{+1} \quad i
$$

The validity problem in interpreted over any class of ordered structures with an infinitely ascending sequence is r.e.-hard.

The validity problem in interpreted over any class of Dedekind complete ordered structures having an infinitely ascending sequence is $\Pi_{1}^{1}$-hard.

unboundedly ascending sequences

$n$

n

The validity problem in interpreted over any class of Dedekind complete ordered structures having unboundedly ascending sequences is co-r.e. hard.

geometrical

$$
\langle D,<\times\langle D,<\quad\langle D,<
$$

$\begin{array}{lll}3 \phi & \langle B \phi & \langle\bar{b} \\ 3 \phi & \langle\bar{B} \phi & \phi \\ 3 \phi & \langle E \phi & \phi \\ 3 \phi & \langle\bar{E} \phi & \phi\end{array}$


$3 \phi \quad 3 \phi \vee \phi \vee 3 \phi \phi$

$3 \phi \quad 3 \phi \vee \phi \vee 3 \phi \phi$

3

333

(A-HS)

$\mathbb{Q}$

(A-HS1)

(A-HS2a) $2(p \rightarrow q) \rightarrow(2 p \rightarrow 2 q)$

(A-HS2b) $2(p \rightarrow q) \rightarrow(2 p \rightarrow 2 q)$

(A-HS3a) $33 p \rightarrow 3 p$

(A-HS3b) $33 p \rightarrow 3 p$

(A-HS4a) $32 p \rightarrow p$

(A-HS4b) $32 p \rightarrow p$

(A-HS5) $3 \uparrow \rightarrow 32 \perp$

(A-HS6) $2 \perp \rightarrow 2-\perp$

(A-HS7a) $33 p \rightarrow 33 p$

(A-HS7b) $33-p \leftrightarrow 3-3 p$

(A-HS7c) $33 p \rightarrow 33 p$

(A-HS8) $(3 p \wedge 3 q) \rightarrow[3(p \wedge 3 q) \vee 3(p \wedge q) \vee 3(3 p \wedge q)]$

$2,2,2-$

$z$

$$
\frac{\operatorname{hor}(p) \rightarrow \phi}{\phi} \frac{\operatorname{ver}(q) \rightarrow \psi}{\psi},
$$

$p, q \quad \phi, \psi$

$\operatorname{hor}(\phi) \quad \phi \wedge 2 \phi \wedge 2-\phi \wedge 2(\phi \wedge 2 \quad \phi \wedge 2-\phi) \wedge 2(\phi \wedge 2 \quad \phi \wedge 2-\phi)$

$\operatorname{ver}(\phi) \quad \phi \wedge 2 \phi \wedge 2 \phi \wedge 2(\phi \wedge 2 \quad \phi \wedge 2 \quad \phi) \wedge 2(\phi \wedge 2 \phi \wedge 2 \phi)$ 


$$
\begin{array}{lccc}
\operatorname{hor}(\phi) & {\left[d_{0}, d_{1}\right]} & \phi & {\left[d_{2}, d_{1}\right]} \\
d_{2} \leq d_{1} & \operatorname{ver}(\phi) & \phi
\end{array}
$$

The axiomatic system $(\boldsymbol{A}-\mathbf{H S})$ is sound and complete for the class of all non-strict interval structures.

A sound and complete axiomatic system for the class of discrete structures can be obtained from (A-HS) by adding the following axiom:

(A-HS ) di screte.

A sound and complete axiomatic system for the class of linear structures can be obtained from (A-HS) by replacing axiom (A-HS8) by the following axiom:

$(A-H S \quad)(33 p) \rightarrow(3 p \vee p \vee 3 p),(33 p) \rightarrow(3 p \vee p \vee 3 p)$.

A sound and complete axiomatic system for $\mathbb{Q}$ can be obtained from the system for the class of linear structures by adding the following axiom:

(A-HS ${ }^{\mathrm{Q}}$ ) $3 \top \wedge 3 \top \wedge$ dense.

$\bar{B} \quad \bar{E} \quad \bar{A}$

$\bar{D} \quad \bar{D}$

$\overline{\mathrm{D}}$

\subsection{Interval logics with binary operators}

The chop operator and (Local) Propositional Interval Logics.

chop $\quad C$

C

$\pi$

$$
\phi::=p|\pi| \phi|\phi \wedge \psi| \phi C \psi
$$

$\langle B \quad\langle E$

$\langle B \phi \quad \phi C \pi$

$\langle E \phi \quad \pi C \phi$

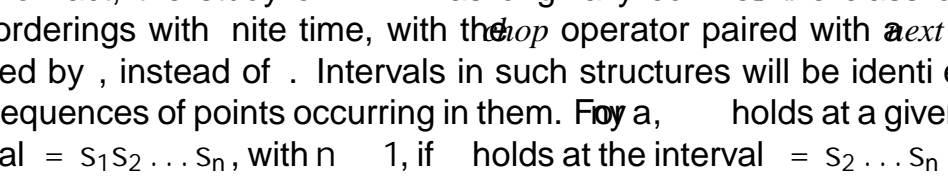


$\pi$

next

$\phi \bigcirc \phi \quad l 1 C \phi$

The satisfiability problem for interpreted over the class of non-strict discrete structures is undecidable.

$G_{1} \quad G_{2}$

The satisfiability problem for -formulas interpreted over the class of (non-strict) dense linear structures is undecidable.

The satisfiability problem for interpreted over the class of (non-strict) linear structures is undecidable.

chop

locality projection principle

(loc-PS1) $\mathbf{M}^{+},\left[d_{0}, d_{1}\right] \quad p \quad p \in V\left(d_{0}\right)$ 
The satisfiability problem for the logic , interpreted over the class of (non-strict) discrete linear structures is (non-elementarily) decidable.

Satisfiability for LPITL is non-elementary.

$\phi \phi^{*}$

chop-star

$\phi$

The following axiomatic system is sound and complete for the class of (non-strict) discrete linear structures:

(A-CLPITL1) enough propositional tautologies;

(A-CLPITL2) $(\phi C \psi) C \xi \leftrightarrow \phi C(\psi C \xi)$;

(A-CLPITL3) $(\phi \vee \psi) C \xi \rightarrow(\phi C \xi) \vee(\psi C \xi)$;

(A-CLPITL4) $\xi C(\phi \vee \psi) \rightarrow(\xi C \phi) \vee(\xi C \psi)$;

(A-CLPITL5) $\pi C \phi \leftrightarrow \phi$;

(A-CLPITL6) $\phi C \pi \leftrightarrow \phi$;

(A-CLPITL7) $p \rightarrow(p C \top)$, with $p \in \mathcal{A P}$;

(A-CLPITL8) $\quad(\quad(\phi \rightarrow \psi) C \top) \wedge(\top C(\xi \rightarrow \chi)) \rightarrow((\phi C \xi) \rightarrow(\psi C \chi))$;

(A-CLPITL9) $\bigcirc \phi \rightarrow \bigcirc \phi ;$

(A-CLPITLQ $) \wedge \wedge(\top C(\phi \rightarrow \bigcirc \phi)) \rightarrow(\top C \phi)$;

(A-CLPITL1 ) $\phi^{*} \leftrightarrow \pi \vee(\phi \wedge \bigcirc \top) C \phi^{*}$,

together with Modus Ponens and the following inference rules:

$$
\frac{\phi}{(\top C \phi)}, \quad \frac{\phi}{(\phi C \top)} \text {. }
$$


projection

$\phi$

projected formula

proj $\phi, \psi \quad \phi \operatorname{proj} \psi$

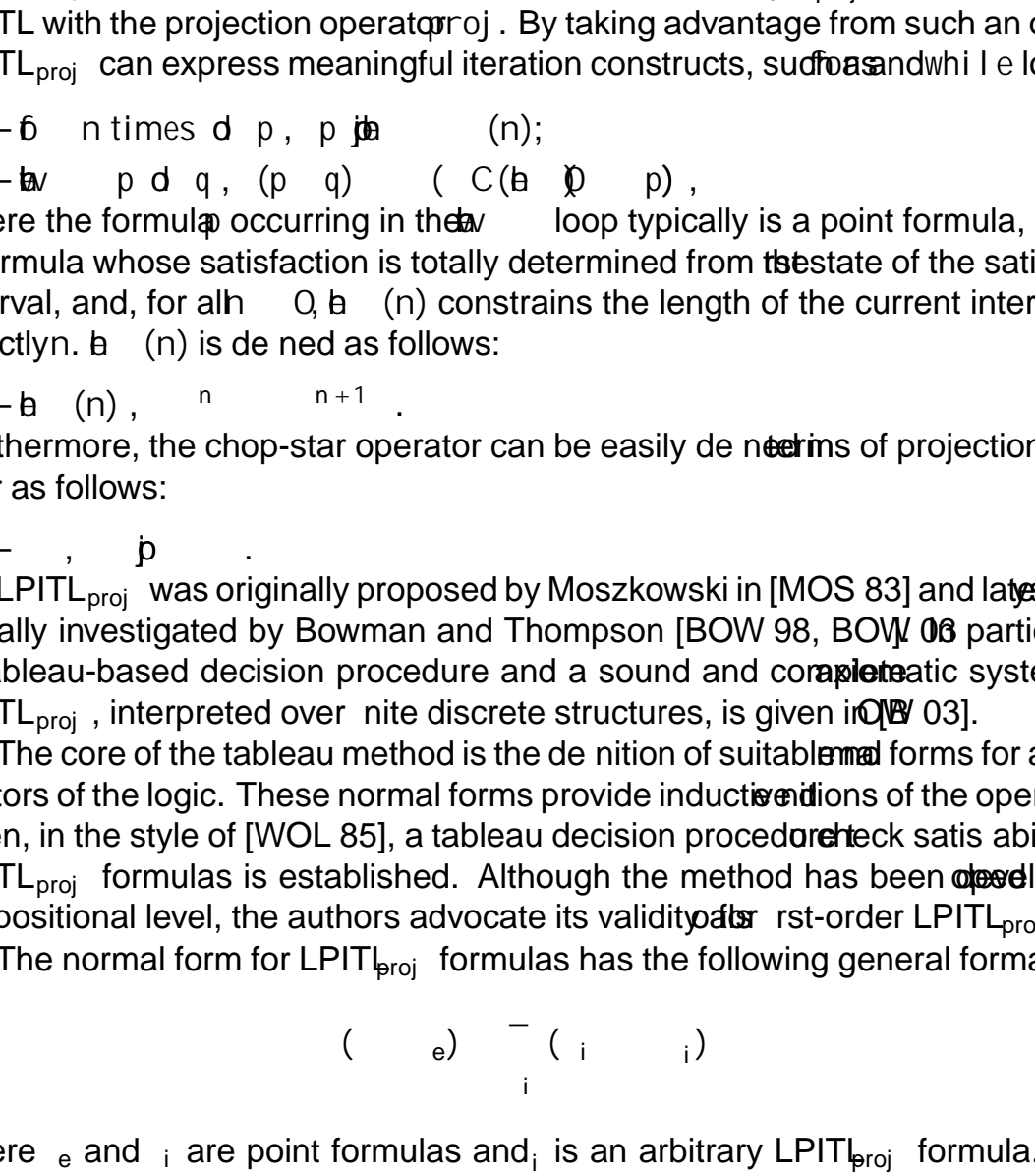

proj 


\section{(A-LPITL1)}

(A-LPITL2) $\quad \pi \leftrightarrow \bigcirc \top$

(A-LPITL3) $\bigcirc \phi \rightarrow \bigcirc \phi$

(A-LPITL4) $\bigcirc(\phi \rightarrow \psi) \rightarrow \bigcirc \phi \rightarrow \bigcirc \psi$

(A-LPITL5) $(\bigcirc \phi) C \psi \leftrightarrow \bigcirc(\phi C \psi)$

(A-LPITL6) $(\phi \vee \psi) C \xi \leftrightarrow \phi C \xi \vee \psi C \xi$

(A-LPITL7) $\phi C(\psi \vee \xi) \leftrightarrow \phi C \psi \vee \phi C \xi$

(A-LPITL8) $\phi C(\psi C \xi) \leftrightarrow(\phi C \psi) C \xi$

(A-LPITL9) $(p \wedge \phi) C \psi \leftrightarrow p \wedge(\phi C \psi), \quad p \in \mathcal{A P}$

(A-LPITL10) $\pi C \phi \leftrightarrow \phi C \pi \leftrightarrow \phi$

(A-LPITL11) $\phi \operatorname{proj} \pi \leftrightarrow \pi$

(A-LPITL12) $\phi \operatorname{proj}(\psi \vee \xi) \leftrightarrow(\phi \operatorname{proj} \psi) \vee(\phi \operatorname{proj} \xi)$

(A-LPITL13) $\phi \operatorname{proj}(p \wedge \psi) \leftrightarrow p \wedge(\phi \operatorname{proj} \psi)$

(A-LPITL14) $\phi \operatorname{proj} \bigcirc \psi \leftrightarrow(\phi \wedge \pi) C(\phi \operatorname{proj} \psi)$

$$
\frac{\phi \rightarrow \bigcirc \phi}{\phi} .
$$

The above axiomatic system is sound and complete for the class of (non-strict) discrete structures.

projection 
The logics $\mathrm{DT}$ and $\mathrm{BDT}+$

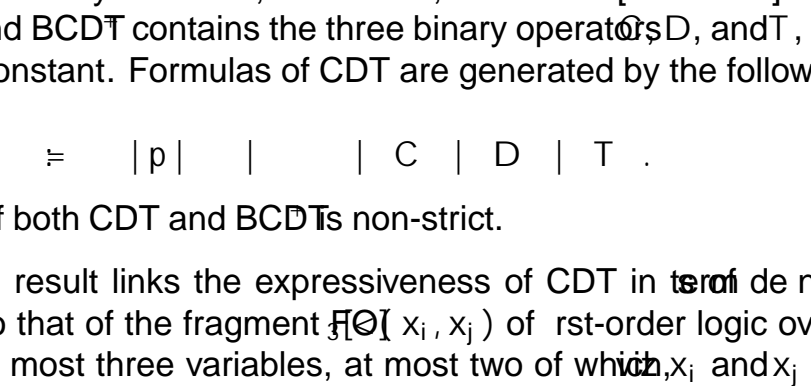

Every binary modal operator definable in $\mathrm{FO}_{3}[<](x, x)$ has an equivalent in , and vice versa.

$$
\begin{aligned}
& 3-=(\pi) C \phi \\
& 3 \phi=(\pi) D \phi \\
& 3 \phi=(\pi) T \phi \\
& 3 \phi=\phi C(\pi)
\end{aligned}
$$

$$
\operatorname{hor}(\phi)
$$

$$
\text { T D }
$$

\section{(A-CDT1)}

(A-CDT2a) $(\phi \vee \psi) C \xi \leftrightarrow \phi C \xi \vee \psi C \xi$

(A-CDT2b) $(\phi \vee \psi) T \xi \leftrightarrow \phi T \xi \vee \psi T \xi$

(A-CDT2c) $\phi T(\psi \vee \xi) \leftrightarrow \phi T \psi \vee \phi T \xi$

(A-CDT3a) $\quad(\phi T \psi) C \phi \rightarrow \psi$

(A-CDT3b) $\quad(\phi T \psi) D \psi \rightarrow \phi$

(A-CDT3c) $\phi T(\psi C \phi) \rightarrow \psi$

(A-CDT4) $\quad \pi C \top \leftrightarrow \pi$ 
(A-CDT5a) $\pi C \phi \leftrightarrow \phi$

(A-CDT5b) $\pi T \phi \leftrightarrow \phi$

(A-CDT5c) $\phi T \pi \rightarrow \phi$

(A-CDT6) $[(\pi \wedge \phi) C \top \wedge((\pi \wedge \psi) C \top) C \top] \rightarrow(\pi \wedge \psi) C \top$

(A-CDT6a) $(\phi C \psi) C \xi \leftrightarrow \phi C(\psi C \xi)$

(A-CDT6b) $\phi T(\psi C \xi) \leftrightarrow(\psi C(\phi T \xi) \vee(\xi T \phi) T \psi)$

(A-CDT6c) $\psi C(\phi T \xi) \rightarrow \phi T(\psi C \xi)$

(A-CDT7d) $(\phi T \psi) C \xi \rightarrow((\xi D \phi) T \psi \vee \psi C(\phi D \xi))$

$$
\begin{gathered}
\frac{\phi}{(\phi C \psi)}, \quad \frac{\phi}{(\phi T \psi)}, \frac{\phi}{(\psi T \phi)}, \\
p \in \mathcal{A P} \quad p \\
\frac{\text { h6 } p) \rightarrow \phi}{\phi} .
\end{gathered}
$$

The above axiomatic system is sound and complete for the class of (non-strict) linear orderings.

A sound and complete axiomatic system for the class of (non-strict) dense linear orderings can be obtained from the system for the class of (non-strict) linear orderings by adding the following axiom:

(A-CDT ) $\pi \rightarrow\left(\begin{array}{lll}\pi C & \pi\end{array}\right)$.

A sound and complete axiomatic system for the class of (non-strict) discrete linear orderings can be obtained from the system for the class of (non-strict) linear orderings by adding the following axiom:

(A-CDT ) $\pi \vee((l 1 C \top) \wedge(T C l)$;

A sound and complete axiomatic system for $\mathbb{Q}$ can be obtained from the system for the class of (non-strict) linear orderings by adding the following axiom:

$\left(\mathbf{A}-\mathbf{C D T}^{\mathrm{Q}}\right)(\pi \rightarrow(\pi C \pi)) \wedge(\pi T \top) \wedge(\pi D \top)$. 
3.3. Restricted interval logics: split logics

$\bar{B} \quad \bar{E}$

split structures

split models

$\phi::=p|\phi \wedge \phi| \quad \phi \mid\langle D \phi|\langle\bar{D} \phi|\langle F \phi|\langle\bar{F} \phi|\phi C \phi| \phi D \phi| \phi T \phi$.

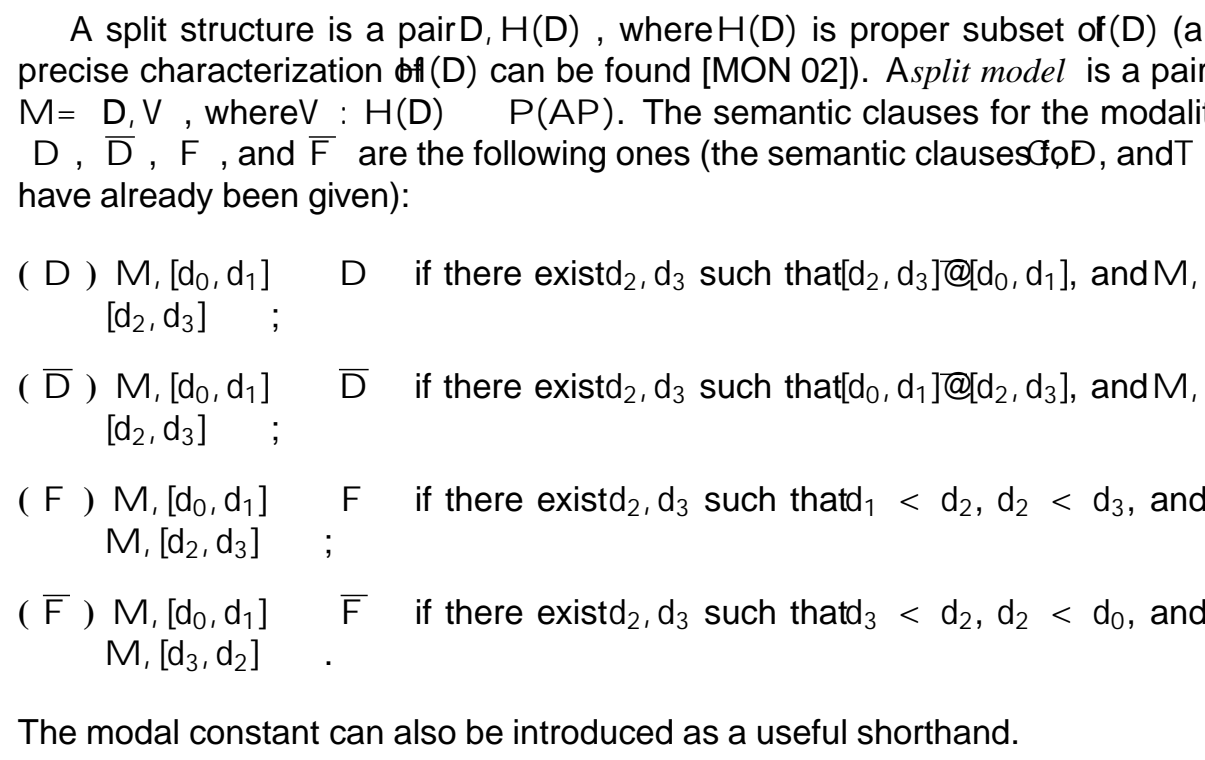




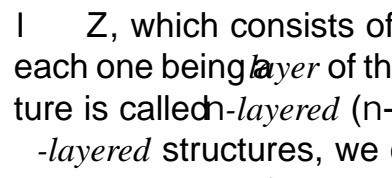

$$
\bigcup \in T
$$
$T$ $n$ $\omega$-layered

upward unbounded
2

$n$

[U $T$,

$x<_{1}$

$y$ $\left[d_{2}, d_{3}\right] \in \mathbf{H}(\mathbb{D})$

$\left[d_{2}, d_{3} \Subset\left[d_{4}, d_{5}\right]\right.$ 
1) interpreted over the class of bounded below, unbounded above, dense, and with maximal intervals split structures can be embedded into

$\left[\bigcup T,<_{1},<_{2}, \downarrow_{0}, \downarrow_{1}\right]$ interpreted over the 2-refinable ;

2) interpreted over the class of bounded below, unbounded above, discrete, and with maximal intervals split structures can be embedded into $\left[\bigcup T,<_{1},<_{2}, \downarrow_{0}, \downarrow_{1}\right]$ interpreted over the 2-refinable $n$ - ;

3) interpreted over the class of bounded below, unbounded above, discrete split structures can be embedded into $\quad\left[\cup T,<_{2}, \downarrow_{0}, \downarrow_{1}\right]$ interpreted over the 2-refinable

The satisfiability problem for formulas, interpreted over the above classes of split structures, is decidable.

\section{A general tableau method for propositional interval logics}

finite tree

$$
\begin{aligned}
& \text { root }
\end{aligned}
$$

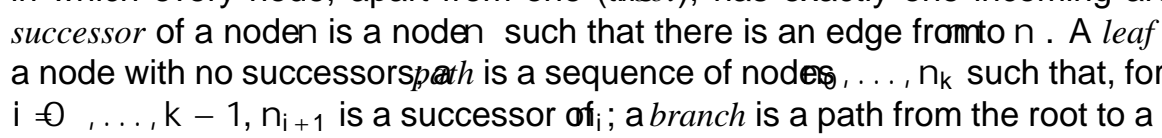

$$
\begin{aligned}
& \text { height } \\
& \mathbf{n}, \mathbf{n} \\
& \mathbf{n} \\
& \mathbf{n}^{\prime} \\
& \mathbf{n} \prec \mathbf{n}^{\prime} \\
& \mathbb{C}=\langle C,< \\
& (\phi,[c, c]) \\
& \mathbf{n} \\
& \mathbb{C} \\
& \text { local flag function } \\
& \text { n } \\
& \phi \in \quad+\quad[c, c] \in \mathbb{I}(\mathbb{C})^{+} \\
& \text {n } \\
& \mathbf{n} \\
& \text { decoration } \nu(\mathbf{n}) \quad((\phi,[c, c]), \mathbb{C}, u) \\
& (\phi,[c, c]) \\
& {[c, c]} \\
& \text { global flag function } \\
& \mathbf{n} \\
& c<c+1<\cdots<c \\
& \mathbb{C}=\{c<c, c<c, \ldots\} \quad \text { decorated } \\
& \nu(\mathbf{n})
\end{aligned}
$$




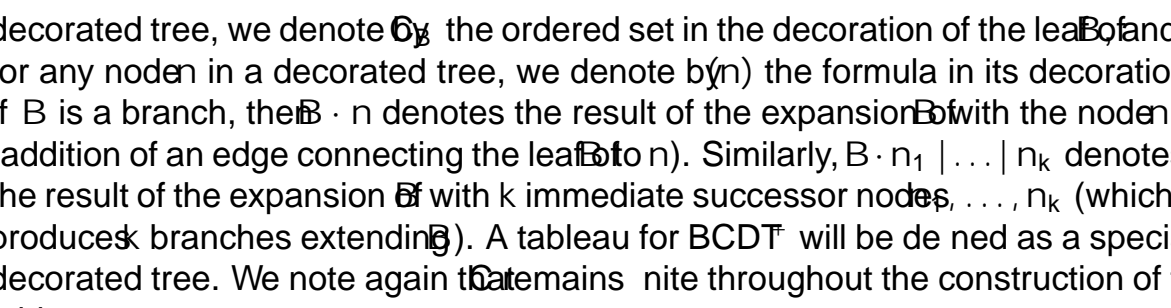

Given a decorated tree $\mathcal{T}$, a branch $B$ in $\mathcal{T}$, and a node $\mathbf{n} \in B$ such that $\nu(\mathbf{n})=((\phi,[c, c]), \mathbb{C}, u)$, with $u(\mathbf{n}, B)=0$, the for $B$ and $\mathbf{n}$ is defined as follows (in all the considered cases, $u\left(\mathbf{n}^{\prime}, B^{\prime}\right)=0$ for all new pairs (n', $\left.B^{\prime}\right)$ of nodes and branches).

- If $\phi=\psi$, then expand the branch to $B \cdot \mathbf{n}$, with $\nu(\mathbf{n})=((\psi,[c, c])$, $\mathbb{C}, u)$.

- If $\phi=\psi_{0} \wedge \psi_{1}$, then expand the branch to $B \cdot \mathbf{n} \cdot \mathbf{n}$, with $\nu(\mathbf{n})=$ $\left(\left(\psi_{0},[c, c]\right), \mathbb{C}, u\right)$ and $\nu(\mathbf{n})=\left(\left(\psi_{1},[c, c]\right), \mathbb{C}, u\right)$.

- If $\phi=\left(\psi_{0} \wedge \psi_{1}\right)$, then expand the branch to $B \cdot \mathbf{n} \mid \mathbf{n}$, with $\nu(\mathbf{n})$ $=\left(\left(\psi_{0},[c, c]\right), \mathbb{C}, u\right)$ and $\nu(\mathbf{n})=\left(\left(\psi_{1},[c, c]\right), \mathbb{C}, u\right)$.

- If $\phi=\left(\psi_{0} C \psi_{1}\right)$ and $c$ is the least element of $\mathbb{C}$, with $c \leq c \leq c$, which has not been used yet to expand the node $\mathbf{n}$ on $B$, then expand the branch to $B \cdot \mathbf{n} \mid \mathbf{n}$, with $\nu(\mathbf{n})=\left(\left(\psi_{0},[c, c]\right), \mathbb{C}, u\right)$ and $\nu(\mathbf{n})=\left(\left(\psi_{1},[c, c]\right), \mathbb{C}, u\right)$.

- If $\phi=\left(\psi_{0} D \psi_{1}\right)$, $c$ is a minimal element of $\mathbb{C} \quad$ such that $c \leq c$, and there exists $c^{\prime} \in[c, c]$ which has not been used yet to expand the node $\mathbf{n}$ on $B$, then take the least such $c^{\prime} \in[c, c]$ and expand the branch to $B \cdot \mathbf{n} \mid \mathbf{n}$, with $\nu(\mathbf{n})$ $=\left(\left(\psi_{0},\left[c^{\prime}, c\right]\right), \mathbb{C}, u\right)$ and $\nu(\mathbf{n})=\left(\left(\psi_{1},\left[c^{\prime}, c\right]\right), \mathbb{C}, u\right)$.

- If $\phi=\left(\psi_{0} T \psi_{1}\right), c$ is a maximal element of $\mathbb{C}$ such that $c \leq c$, and there exists $c^{\prime} \in[c, c]$ which has not been used yet to expand the node $\mathbf{n}$ on $B$, then take the greatest such $c^{\prime} \in[c, c]$ and expand the branch to $B \cdot \mathbf{n} \mid \mathbf{n}$, so that $\nu(\mathbf{n})$ $=\left(\left(\psi_{0},\left[c, c^{\prime}\right]\right), \mathbb{C}, u\right)$ and $\nu(\mathbf{n})=\left(\left(\psi_{1},\left[c, c^{\prime}\right]\right), \mathbb{C}, u\right)$.

- If $\phi=\left(\psi_{0} C \psi_{1}\right)$, then expand the branch to $B \cdot(\mathbf{n} \cdot \mathbf{m})|\ldots|(\mathbf{n} \cdot \mathbf{m}) \mid\left(\mathbf{n}^{\prime} \cdot\right.$ $\left.\mathbf{m}^{\prime}\right)|\ldots|\left(\mathbf{n}_{-}^{\prime} \cdot \mathbf{m}_{-}^{\prime}\right)$, where:

1) for all $c \in[c, c], \nu(\mathbf{n})=\left(\left(\psi_{0},[c, c]\right), \mathbb{C}, u\right)$ and $\nu(\mathbf{m})=\left(\left(\psi_{1}\right.\right.$, $[c, c]), \mathbb{C}, u)$;

2) for all $i \leq k \leq j-1$, let $\mathbb{C}$ be the interval structure obtained by inserting a new element $c$ between $c$ and $c+1$ in $[c, c], \nu\left(\mathbf{n}^{\prime}\right)=\left(\left(\psi_{0},[c, c]\right), \mathbb{C}, u\right)$, and $\nu\left(\mathbf{m}^{\prime}\right)=\left(\left(\psi_{1},[c, c]\right), \mathbb{C}, u\right)$.

- If $\phi=\left(\psi_{0} D \psi_{1}\right)$, then repeatedly expand the current branch, once for each minimal element $c$ (where $[c, c]=\left\{c=c_{0}<c_{1}<\cdots c\right\}$ ), by adding the decorated sub-tree $(\mathbf{n} \cdot \mathbf{m})|\ldots|(\mathbf{n} \cdot \mathbf{m})\left|\left(\mathbf{n}^{\prime} \cdot \mathbf{m}^{\prime}\right)\right| \ldots\left|\left(\mathbf{n}^{\prime} \cdot \mathbf{m}^{\prime}\right)\right|\left(\mathbf{n}^{\prime \prime} \cdot \mathbf{m}^{\prime \prime}\right)|\ldots|\left(\mathbf{n}^{\prime \prime} \cdot \mathbf{m}^{\prime \prime}\right)$ to its leaf, where: 
1) for all $c$ such that $c \in[c, c], \nu(\mathbf{n})=\left(\left(\psi_{0},[c, c]\right), \mathbb{C}, u\right)$ and $\nu(\mathbf{m})$ $=\left(\left(\psi_{1},[c, c]\right), \mathbb{C}, u\right)$;

2) for all $0<k \leq i$, let $\mathbb{C}$ be the interval structure obtained by inserting a new element $c^{\prime}$ immediately before $c$ in $[c, c]$ and $\nu\left(\mathbf{n}^{\prime}\right)=\left(\left(\psi_{0},\left[c^{\prime}, c\right]\right), \mathbb{C}, u\right)$ and $\nu\left(\mathbf{m}^{\prime}\right)=\left(\left(\psi_{1},\left[c^{\prime}, c\right]\right), \mathbb{C}, u\right)$;

3) for all $0 \leq k \leq i$, let $\mathbb{C}$ be the interval structure obtained by inserting $a$ new element $c^{\prime}$ in $\mathbb{C}$, with $c^{\prime}<c$, which is incomparable with all existing predecessors of $c, \nu\left(\mathbf{n}^{\prime \prime}\right)=\left(\left(\psi_{0},\left[c^{\prime}, c\right]\right), \mathbb{C}, u\right)$, and $\nu\left(\mathbf{m}^{\prime \prime}\right)=\left(\left(\psi_{1},\left[c^{\prime}, c\right]\right), \mathbb{C}, u\right)$.

- If $\phi=\left(\psi_{0} T \psi_{1}\right)$, then repeatedly expand the current branch, once for each maximal element $c$ (where $[c, c]=\{c<c+1<\cdots c=c\}$ ), by adding the decorated sub-tree $(\mathbf{n} \cdot \mathbf{m})|\ldots|(\mathbf{n} \cdot \mathbf{m})\left|\left(\mathbf{n}^{\prime} \cdot \mathbf{m}^{\prime}\right)\right| \ldots\left|\left(\mathbf{n}_{-}^{\prime} \cdot \mathbf{m}_{-}^{\prime}\right)\right|\left(\mathbf{n}^{\prime \prime} \cdot \mathbf{m}^{\prime \prime}\right)|\ldots|\left(\mathbf{n}^{\prime \prime} \cdot \mathbf{m}^{\prime \prime}\right)$ to its leaf, where:

1) for all $c$ such that $c \in[c, c] \nu(\mathbf{n})=\left(\left(\psi_{0},[c, c]\right), \mathbb{C}\right.$, u) and $\nu(\mathbf{m})$ $=\left(\left(\psi_{1},[c, c]\right), \mathbb{C}, u\right)$;

2) for all $j \leq k<n$, let $\mathbb{C}$ be the interval structure obtained by inserting a new element $c^{\prime}$ immediately after $c$ in $[c, c]$ and $\nu\left(\mathbf{n}^{\prime}\right)=\left(\left(\psi_{0},\left[c, c^{\prime}\right]\right), \mathbb{C}, u\right)$ and $\nu\left(\mathbf{m}^{\prime}\right)=\left(\left(\psi_{1},\left[c, c^{\prime}\right]\right), \mathbb{C}, u\right)$;

3) for all $j \leq k \leq n$, let $\mathbb{C}$ be the interval structure obtained by inserting a new element $c^{\prime}$ in $\mathbb{C}$, with $c<c^{\prime}$, which is incomparable with all existing successors of $c, \nu\left(\mathbf{n}^{\prime \prime}\right)=\left(\left(\psi_{0},\left[c, c^{\prime}\right]\right), \mathbb{C}, u\right)$, and $\nu\left(\mathbf{m}^{\prime \prime}\right)=\left(\left(\psi_{1},\left[c, c^{\prime}\right]\right), \mathbb{C}, u\right)$.

Finally, for any node $\mathbf{m}(\neq \mathbf{n})$ in $B$ and any branch $B^{\prime}$ extending $B$, let $u\left(\mathbf{m}, B^{\prime}\right)$ be equal to $u(\mathbf{m}, B)$, and for any branch $B^{\prime}$ extending $B, u\left(\mathbf{n}, B^{\prime}\right)=1$, unless $\phi=\left(\psi_{0} C \psi_{1}\right), \phi=\left(\psi_{0} D \psi_{1}\right)$, or $\phi=\left(\psi_{0} T \psi_{1}\right)$ (in such cases $\left.u\left(\mathbf{n}, B^{\prime}\right)=0\right)$.

$$
\psi_{0} C \psi_{1} \quad\left(\psi_{0} C \psi_{1}\right)
$$

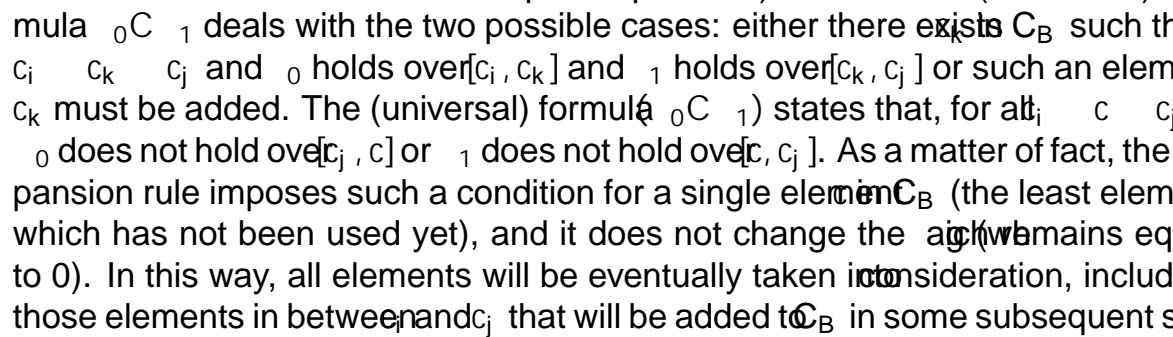

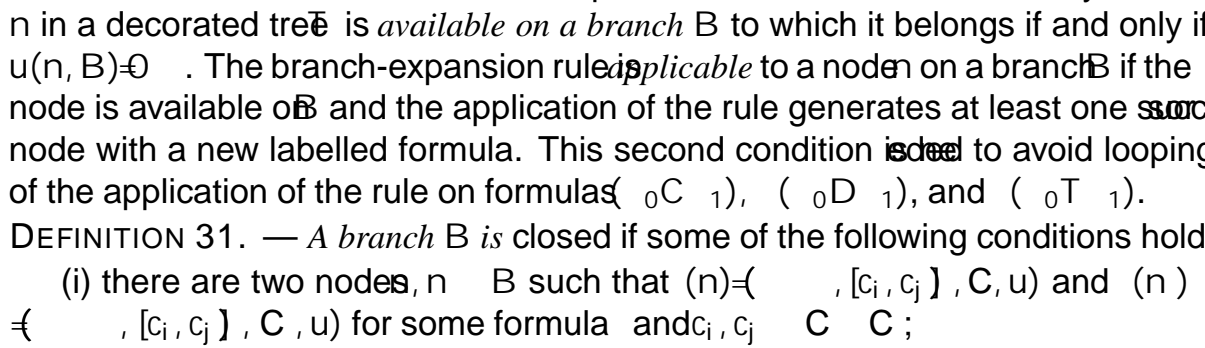




$$
\begin{array}{rrr}
\mathbf{n} & \nu(\mathbf{n})=((\pi,[c, c]), \mathbb{C}, u) \quad c \neq c \\
\mathbf{n} & \nu(\mathbf{n})=((\pi,[c, c]), \mathbb{C}, u) \quad c=c \\
\text { The } & \text { for a branch } B \text { in a decorated }
\end{array}
$$

tree $\mathcal{T}$ is defined as follows:

1) Apply the branch-expansion rule to a branch $B$ only if it is open;

2) If $B$ is open, apply the branch-expansion rule to the closest to the root available node in $B$ for which the branch-expansion rule is applicable.

A for a given formula $\phi \in \quad+$ is any finite decorated tree $\mathcal{T}$ obtained by expanding the three-node decorated tree built up from an empty-decoration root and two leaves with decorations $((\phi,[c, c]),\{c<c\}, u)$ and $((\phi,[c, c]),\{c\}, u)$, where the value of $u$ is 0 , through successive applications of the branch-expansion strategy to the existing branches.

$$
\begin{array}{ccc}
\phi \in & +\mathcal{T}
\end{array} \quad \phi \mathbf{n} \in \mathcal{T}
$$

$\mathcal{T}$ for $\phi$ is closed, then $\phi$ is not satisfiable. Moreover, if $\phi \in++$ is a valid formula then there is a closed tableau for $\phi$.

\section{First-Order Interval Logics and Duration Calculi}

\subsection{The logic}

\section{C}

$$
\begin{aligned}
& \text { global rigid temporal flexible } \theta_{1}, \ldots, \theta \\
& p, q, \ldots \quad p \quad i q \quad j \\
& \phi::=\theta\left|p\left(\theta_{1}, \ldots, \theta\right)\right| \exists x \phi|\quad \phi| \phi \wedge \psi|\bigcirc \phi| \phi C \psi
\end{aligned}
$$




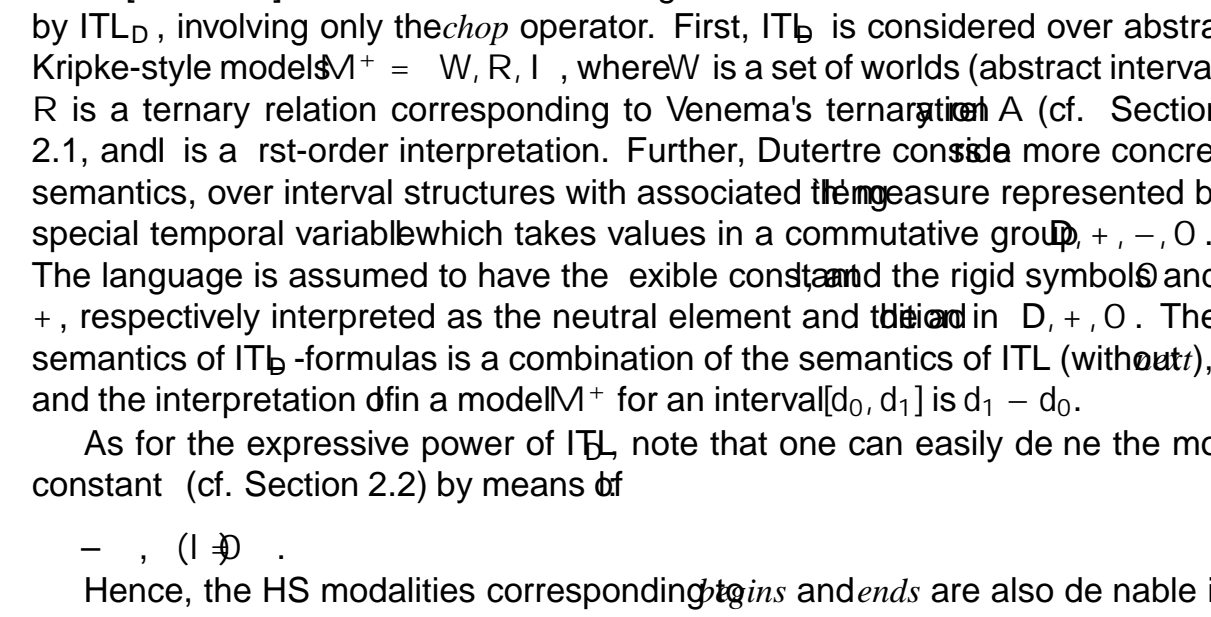

(A-ITL1) $(\phi C \psi) \wedge(\phi C \xi) \rightarrow \phi C(\psi \wedge \xi)$

(A-ITL2) $(\phi C \psi) \wedge(\xi C \psi) \rightarrow(\phi \wedge \xi) C \psi$

(A-ITL3) $((\phi C \psi) C \xi) \leftrightarrow(\phi C(\psi C \xi))$

(A-ITL4) $(\phi C \psi) \rightarrow \phi \quad \phi$

(A-ITL5) $(\phi C \psi) \rightarrow \psi \quad \psi$

(A-ITL6) $((\exists x) \phi C \psi) \rightarrow(\exists x)(\phi C \psi) \quad x \quad \psi$

(A-ITL7) $(\phi C(\exists x) \psi) \rightarrow(\exists x)(\phi C \psi) \quad x \quad \phi$

(A-ITL8) $((l=x) C \phi) \rightarrow \quad((l=x) C \quad \phi)$

(A-ITL9) $(\phi C(l=x)) \rightarrow(\phi C(l=x))$

(A-ITL10) $(l=x+y) \leftrightarrow((l=x) C(l=y))$

(A-ITL11) $\phi \rightarrow(\phi C(l=0))$

(A-ITL12) $\phi \rightarrow((l=0) C \phi)$ 
$\frac{\phi \rightarrow \psi}{\phi C \xi \rightarrow \psi C \xi}$,

Some extensions and variations of ITL

direction

signed intervals

5.2. The logic $N L$

contracting

left right

neighbourhood

logic 


$$
\begin{gathered}
\diamond \diamond \\
\phi::=\theta\left|p\left(\theta_{1}, \ldots, \theta\right)\right| \phi|\phi \wedge \psi| \diamond \phi|\diamond \phi| \exists x \phi, \\
\theta_{1}, \ldots, \theta \quad \diamond \quad \diamond
\end{gathered}
$$

\section{$\mathcal{A}$-models}

chop

$\phi C \psi \quad \exists x, y(l=x+y) \wedge \diamond \diamond((l=x) \wedge \phi \wedge \diamond((l=y) \wedge \psi))$

$\diamond \diamond \diamond \diamond \quad \diamond \quad \nabla \quad \diamond \quad \diamond$

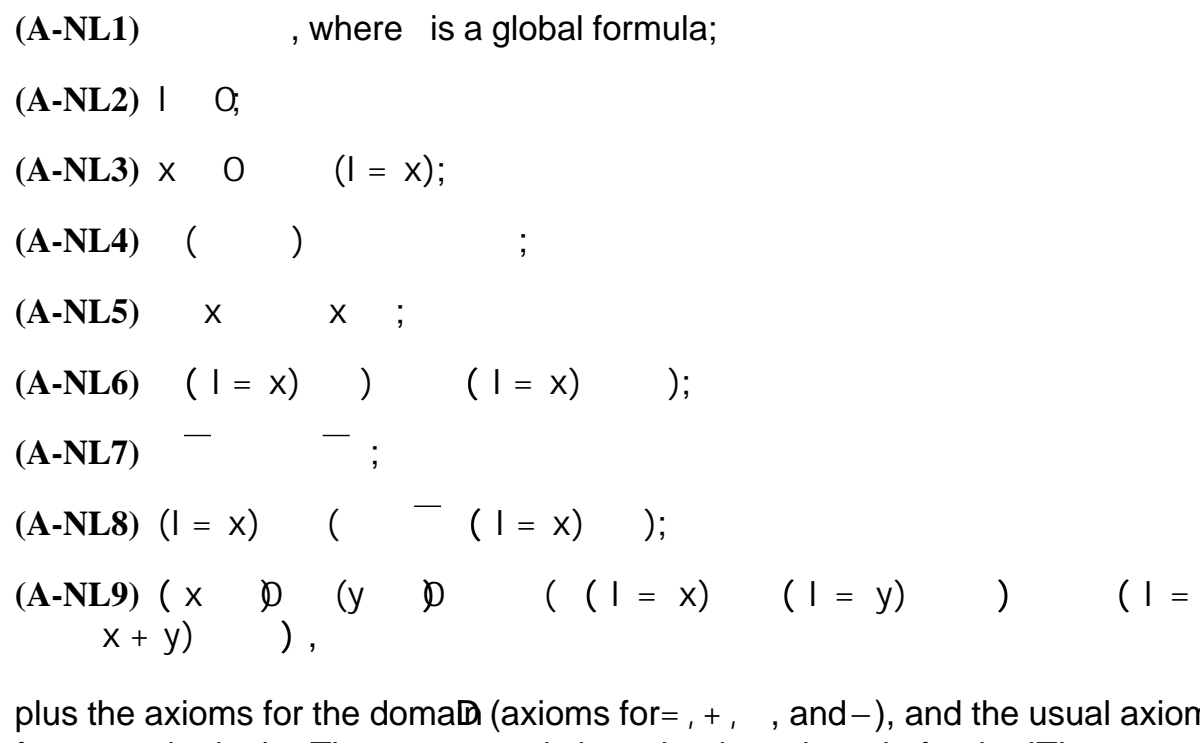




\subsection{Duration calculi}

state

duration

The calculus DC/ITL

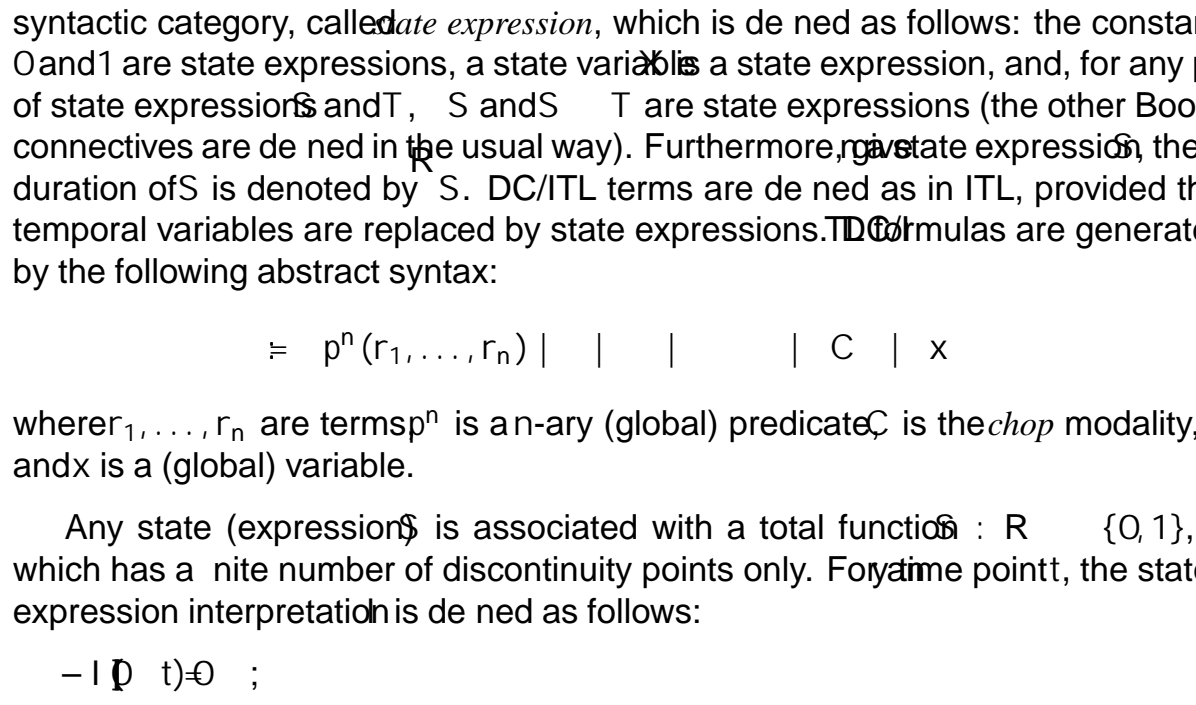




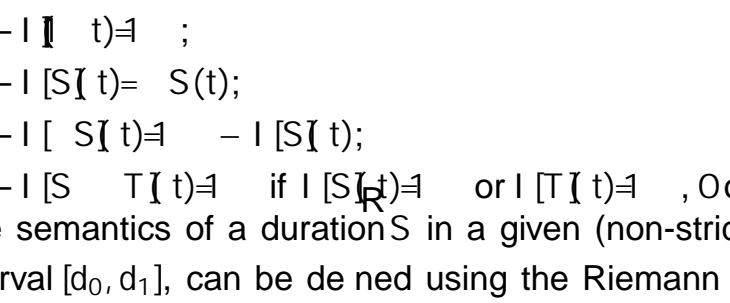

$S$

$\int 1$

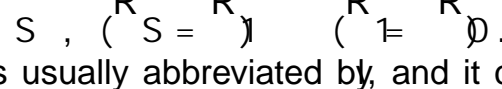

$\int_{0}^{1} \mathcal{I}[S](t) d t$

$$
l=0
$$

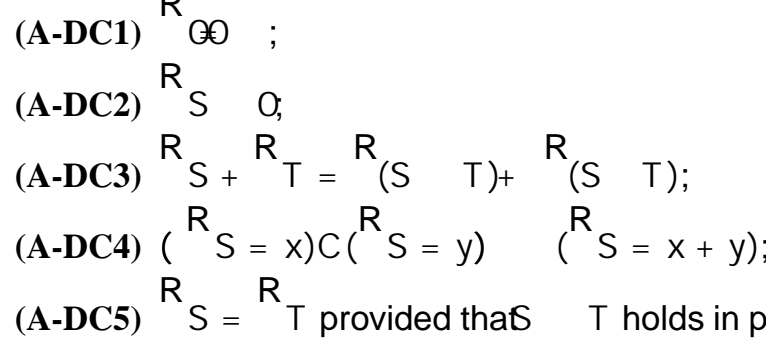

$$
S_{1} \ldots S \quad \quad \bigvee={ }_{=1} S \leftrightarrow
$$

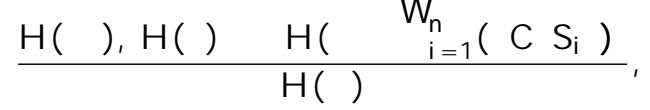

$$
\text { chop } \quad \begin{array}{ll}
H(\phi) \\
\end{array} \quad X^{H} \quad \phi
$$

$H(X)$

$$
\begin{aligned}
& \operatorname{over} \mathbb{N} \\
& \operatorname{over} \mathbb{Q}
\end{aligned}
$$




$$
\begin{aligned}
& \mathbb{N} \mathbb{Q} \quad \mathbb{R} \\
& l=k \\
& \lceil S\rceil \\
& \int S=\int T \\
& \lceil S\rceil \\
& \mathbb{N} \\
& \mathbb{R} \\
& \phi::=\top|\perp| l P k|I=0| I=l|\phi \vee \psi| \phi \wedge \psi \mid \phi C \psi, \\
& k \\
& P \in\{<, \leq,=, \quad,>\} \quad I \quad \int S
\end{aligned}
$$

Some extensions and variations of Duration Calculus

$\lceil S\rceil$

$$
l=k
$$


6. Summary and concluding remarks

$\mathcal{P N} \mathcal{L}$ 


\section{References}

\section{LNCS \\ Proc. of 9th International Conference on Automated Deduction}

Proc. of TAPSOFT

of the ACM

Communications

national Joint Conference on Artificial Intelligence

Proc. of the 9th Inter-

nal of Logic and Computation

Jour-

Journal of Logic and Computation

11th International Joint Conference on Artificial Intelligence

Proc. of the

The Logic of Time (2nd Edition)

Tracts in Theoretical Computer Science

Modal Logic

Cambridge

Proc. of the International Conference Tableaux 1998

Applied Logic

Advances in Temporal Logic

Journal of Logic and Computation 
Formal Logic

of the 8th International Conference on Computer Aided Verification

Proc.

SIAM Journal on Computing

Processing Letters

Information

\begin{abstract}
Processing Letters
\end{abstract}
puter Science

Proc. of the 10th Symposium on Theoretical Aspects of Com-

\author{
Hybrid Systems
}

A Classical Mind: Essays in Honour of C.A.R. Hoare

Fundamentals of Computation Theory

\title{
Difference
}

Compositionality: the Significant

Proc. of the Annual Conference of the European Association for Computer Science Logic

Time Systems

Duration Calculus. A Formal Approach to Real-

Journal of Logic and Computation

Annals of Mathematics and Artificial Intelli-

gence

Proc. of the 14th

International Conference on Software Engineering

Proc. of the 12th Foundations of Software Technology and Theoretical Computer Science LNCS

Proc. of the 13th Conference on 
Software Engineering and Methodology

ACM Transactions on

Fundamenta Informaticae

ral Logic, Bonn, Germany

Proc. of the First InternationalConference on TempoLNCS

Fundamenta Informaticae

Science

Theoretical Computer

Science

oretical Computer Science

The -

Word Meaning and Montague Grammar

Proc. of the 10th International Symposium on Logic in Computer Science

http://ca.informatik.uni-oldenburg.de/ fraenzle/diss.ps.gz

Duration Calculus: A Logical Approach to Real-Time Systems. Workshop proceedings of the 10th European Summer School in Logic, Languages and Information (ESSLLI X), Saarbrücken, Germany, August 1998. Final version to appear in a special issue of BCS FACS, 2004.

Proc. of the International Symposium on Formal Techniques in Real-Time and Fault-Tolerant systems (FTRTFT 2002)

LNCS 
ical Foundations and Computational Aspects

Foundations and Computational Aspects

tificial Intelligence

Logic

2003
Ar-

Journal of Philosophical $r-$

Temporal Logic, vol. 2: Mathematical 
Aspects of Computing

\section{Computation}

of Philosophical Logic

Formal Aspects of Computing

Science $\quad$ ANCS $\quad$ Advances in Computing

Science $\quad$ ANCS $\quad$ Advances in Computing

Introduction to Automata Theory, Languages, and

Journal

Semantics from Different Points of View

the 8th British Colloquium for Theoretical Computer Science

Proceedings of

Executable Modal and Temporal Logics

6th Asian Computing Science Conference

Proc. of

Journal of Symbolic

Logic

Multi-Dimensional Modal Logics

and Computation

Journal of Logic

Logic in Artificial Intelligence 2002

Proc. the European Conference on 
Proc. of the 1983 Workshop on Logics of Programs

Executing Temporal Logic Programs

Programming Concepts, Methods and Calculi

$\begin{array}{lr}\text { Non-Classical Logics } & \text { Journal of Applied } \\ \text { Computation } & \text { Journal of Symbolic }\end{array}$

Significant Difference

Compositionality: the

Proc. of the 27th International Colloquium on Automata, Languages and Programming

Proc. of the 15th Annual IEEE Symposium on Logic in Computer Science LICS 2000

Proc. of the ESSLLI Workshop on Interval Temporal Logics and Duration Calculi

Proc. of the European Conference on Computer Science Logics, Fischbachan, Munich, Germany

Journal of the ACM

Publications of the Research Institute for Mathematical Sciences

Proc.

of the 4th International Conference on Formal Techniques in Real-Time and Fault-Tolerant Systems

23rd Annual Software Engineering Workshop, NASA Goddard 
Information Processing Letters

Fundamenta Informaticae

ence on Computer Science Logic

Proc. of the Annual European Confer-

Proc. of the Annual European Conference on Computer Science Logic CSL'2001

Elements of Symbolic Logic

Journal of Philosophical Logic

First Annual IEEE Symposium on

Logic in Computer Science LICS'86

Proc. of the 11th Advanced Research Working Conference on Correct Hardware Design and Verification Methods CHARME 2001 LNCS

Proc. of the Twenty-Fifth Annual International Computer Software and Applications Conference COMPSAC 2001

Notre

Dame Journal of Formal Logic

Journal of Logic and Com-

putation 
Proc. of the 5th National Conference of the American Association for Artificial Intelligence $(A A A I)$

et Analyse

Journal of Computer Science and Technology 